\title{
CONTRIBUCIÓN AL ESTUDIO DEL PLURAL DE LA DECLINACIÓN TEMÁTICA LATINA
}

\begin{abstract}
The Latin language made partly the plural of the thematic declension on the basis of an alomorphic structure, ${ }^{*}-\bar{o} s,{ }^{*}-o i,{ }^{*}$-ốis, and through processes of agglutination.

There are still traces of all this in Latin and in the Indoeuropean languages.
\end{abstract}

Existe un buen número de testimonios epigráficos latinos en donde podemos ver nominativos del plural temáticos acabados en -es, -is y -eis que plantean una serie de problemas de indole fonética y morfológica por cuanto se desvian de las marcas canónicas tradicionalmente aceptadas tanto para el latín como para las diversas lenguas IE. Así vemos Atilies Saranes CIL I 23 ', uiolaries, rosaries, coronaries 980, coques, magistres 1447, duomuires 1511 , heisce ministris 681 , Vertuleieis, libereis 1531, Q. M. Minucieis $Q$. f. Rufeis 584, filis CIL IX 1705 y otros muchos ejemplos que se espacian desde finales del s. III o comienzos del II a.C. hasta el s. I d.C. ${ }^{2}$.

Según Ernout ${ }^{3}$, si bien estas formas aparecen en muchas ocasiones acompañando a dobles praenomina o bien refiriéndose a dos personajes, no tienen valor dual, sino que se deben a la influencia analógica de los demostrativos seguidos de enclítica tales como his-ce, eis-dem. Estas formas empleadas por Plauto ante palabra que comienza por vocal, no conservan en cambio en las inscripciones tal condicionamiento, sino que junto a eisdem e hisce aparece eis y sobre tal modelo debió crearse el nominativo plural temático en -eis. Posteriormente este mismo autor

\footnotetext{
' Pero cf. P. Poccetti, Nuovi documenti italici, Pisa 1979, p. 150.

2 Cf. F. Sommer, Handbuch der lateinischen Laut- und Formenlehre, Heidelberg 1914, p. 346; A. Ernout, "Le parler de Préneste d'après les inscriptions", $M S L 13$, 1905-1906, pp. 343-346. Geográficamente están ampliamente atestiguados, especialmente en el Lacio, en torno a Roma, asi en Tíbur, Cora, Sora y al Sur en Capua y Benevento. También en la costa Adriática en Ariminum e incluso en Cartagena.

${ }^{3}$ A. Ernout, MSL 13, 1905-1906, p. 345.
} 
piensa que tal vez se trate de una contaminación entre $-e i,-\bar{\imath}$ lat. y $-\bar{o} s$ con $-s$ de otros dialectos itálicos ${ }^{4}$. En términos parecidos a esta última propuesta se manifiestan Devoto ${ }^{5}$ y Monteil ${ }^{6}$. En definitiva, la analogía y la contaminación son los presupuestos teóricos en que se basan las diversas explicaciones, presupuestos que en muchos casos encubren una argumentación ad hoc y adolecen de una base globalizadora. Las teorías basadas en préstamos de morfemas o partes de morfemas de lenguas itálicas tropiezan con objeciones teóricas?.

Nuestra propuesta arranca del Indoeuropeo postanatolio en el que hubieron de existir para el plural de la declinación temática, al menos, tres alomorfos, a saber, ${ }^{*}-\bar{o} s,{ }^{*}-o i,{ }^{*}$-ōis en la incipiente creación del plural. Estos alomorfos, en un primer momento, marcarian simplemente la categoría de pluralidad y posteriormente se les añadirían las desinencias canónicas casuales, si bien en algunos casos aún quedan restos de alomorfismo. Las lenguas particulares posteriormente efectuaron procesos de elección en lo concerniente a la marca de nominativo pl. temático, bien eligiendo solamente ${ }^{*}-o i$, caso del latín, griego y antiguo eslavo, bien *-ōs, caso del osco y umbro, bien especializando ${ }^{*} \bar{o} s$ para los nombres y *-oi para los pronombres, así en antiguo indio y gótico, o bien dentro de la categoría nominal empleando *-oi para el nominativo frente $a^{*}-\bar{o} s$ para el vocativo como hace el antiguo irlandés o sim-

4 A. Ernout, Morphologie historique du latin, París 1953, pp. 31-32, donde reconoce que el origen de tales nominativos es oscuro.

${ }^{3}$ G. Devoto, Storia della lingua di Roma, Bolonia 1944, pp. 192-193, para quien tales nominativos son una contaminación del tipo $-\bar{l}$ y del tipo $-s$ añadiendo: "La portata di questi esempi esce dai limiti prenestini per invadere anche il territorio romano propriamente detto. Da una parte, attraverso gli stretti legami che passano tra forme apparentemente rustiche e forme apparentemente arcaiche, i nominativi ibridi in -eis trovano la strada verso i testi giuridici come già l'aveva trovata la desinenza del genitivo in -os; dall'altra la Roma del III secolo non è soltanto esportatrice di lingua, ma anche importatrice di elementi dialettali stranieri che non intaccano la struttura della lingua quando si tratta di modelli abbastanza lontani come i greci, i gallici o gli etruschi; ma che possono penetrare più profondamente quando si tratti di modelli oschi.»

6 P. Monteil, Éléments de phonétique et de morphologie du latin. París 1973, p. 164, piensa que los nominativos plurales temáticos en -es, -eis tal vez sean consecuencia de un cruce entre -ōs y -oi más $s$; dicha $s$ estaria tomada analógicamente de la flexión atemática y terminaria dando una forma -ois a partir de la cual se explicarian los nominativos plurales temáticos en -es y -eis. También W. Blümel en Untersuchungen zu Lautsystem und Morphologie des vorklassischen Lateins, Munich 1972, p. 58 considera aceptable la adaptación de $-s$ a partir del plural de otros temas, o bien la transferencia de -es de temas pronominales en $-i$.

7 W. Blümel, Untersuchungen.... p. 58: "gegen die Annahme, -s sei durch oskischen Einfluss zu erklären, sind vor allem theoretische Einwände zu erheben; Morphe oder gar Teile von Morphen (die oskische Endung lautet - $\bar{u} s$ ) werden nicht so leicht wie etwa lautliche Besonderheiten von einer Sprache in die andere entlehnt.» 
plemente utilizando indiferentemente ${ }^{*}$-ōs y ${ }^{*}$-oi para el nominativo plural como se atestigua en el celta de la Peninsula Ibérica. Ahora bien, junto a estas dos desinencias propugnamos *-ōis, una hipercaracterización de *-oi más $-s$ pluralizante cuyo rendimiento morfológico fue menor que el de ${ }^{*}-\bar{o} s$ y *-oi y que, sin embargo, puede descubrirse como elemento componencial en el plural de los temáticos de muchas lenguas, como veremos a continuación, y en los restos latinos de los nominativos plurales en -es, -is y -eis.

El tratamiento de ${ }^{*}$-ois en latín sería idéntico al de ${ }^{*}$ ooi, a saber, $*_{-o i s}>-e i s>-e s s-i s^{8}$. En el territorio del Lacio hallamos una serie de inscripciones cuyo nominativo plural acaba en - $o$. Así en una inscripción mutilada leemos Metilio CIL $\mathrm{I}_{2}$ 59, Q. K. Cestio 61, Pomplio 30, Aidicio 2442. Estas formas han sido objeto de diversas explicaciones. Para Sommer ${ }^{9}$ se tratarian de duales como los del griego en $-\omega$. Sin embargo sabemos que en latín no existe la categoria de dual y por ello Ernout pensó que se trataba de nominativos singulares empleados erróneamente en lugar del plural ${ }^{10}$. Según Devoto debemos pensar que están creados sobre el morfema ${ }^{*}-\bar{o} s$ de los modelos osco-umbros ${ }^{11}$.

Lo cierto es que sobre esta desinencia las cosas no están claras. Nosotros creemos que se trata de restos de nominativo plural en -ōs que cedieron ante el morfema -oi. Y esto fue lo que sucedió precisamente en osco-umbro pero en una dirección contraria, es decir, generalizando -ōs

${ }^{\star}$ Cf. C. Juret, Manuel de phonétique latine, Paris 1921, p. 293; F. Sommer, Handbuch..., p. 346; A. Ernout, Morphologie..., p. 30; J. Safarewicz, Historische lateinische Grammatik, Halle 1969, pp. 127-128; M. Bassols, Fonética latina, Madrid 1967, pp. 96-97. El antiguo diptongo IE. *-oi final fue cerrándose paulatinamente, en primer lugar mediante - ei ya en época preliteraria de forma que ejemplos como foideratei CIL I, 581 (año 186 a.C.), seruei 614 (198 a.C.) deben representar más bien la pronunciación cerrada de $\bar{e}=$ [e] que la pronunciación de un diptongo, por cuanto en el s. III tenemos atestiguada la forma ploirume CIL $\mathrm{I}_{2}$ 9. En Paul. Fest. 224, 4 leemos: PILUMNOE POPLOE, in carmine saliari uelut pilis uti assueti, uel quia praecipue pellant hostis. Asimismo podemos ver en Paul. Fest. 76, 16: FEsCEMNOE uocabantur, qui depellere fascinum credebantur. Dicha forma en -oe debe notar también una $\bar{e}$ y no creemos que deba ser corregida por -oi como propugnaba A. Ernout en Morphologie..., p. 31. Lo cierto es que -ei se mantuvo durante mucho tiempo por influencia de los gramáticos. Asi Lucil., Saturarum fragmenta 377-379, propugna la pronunciación $-\bar{l}$ para genitivo singular y -ei para nominativo plural cuando escribe: Iam 'puerei uenere' ' $e$ ' postremum facito atque ' $i$ ' / ut puerei plures fiant; ' $i$ ' si facis solum, / 'pupilli pueri Lucili', hoc unius fiet. Cf. también A. Gelio, Noctes Atticae XII 26,4 , quien se expresa en términos parecidos. Finalmente tenemos $-\bar{l}$ ampliamente atestiguado ya desde el s. II a.C. en las inscripciones, purgatī CIL $\mathrm{I}_{2} 586$ (12) del año 160 a.C., ceterī 584 (29) del año 117 a.C.

9 F. Sommer, Handbuch..., p. 348.

${ }_{10}$ A. Ernout, MSL 13, 1905-1906, p. 346. Cf. también K. Meister, LateinischeGriechische Eigennamen, Leipzig 1916, p. 16 ss.

" G. Devoto, Storia.... p. 192. 
y eliminando -oi. Se nos podría objetar que en latín una final $-s$ no cae tras vocal larga. Sin embargo las formas de gentilicio en $-\bar{o}$ atestiguadas se hallan en una zona perfectamente definida, dos ejemplos en Preneste (Cestio y Metilio), una en Roma (Pomplio) y una en Lanuvio (Aidicio), en la zona norte del Lacio donde $-s$ final cae incluso tras vocal larga como se atestigua en los praenomina prenestinos Maiō < ${ }^{*}$ Maiōs, Minō < ${ }^{*}$ Minōs = lat. Maior, Minor o bien en falisco donde podemos leer $c r \bar{a}<{ }^{*} c r a \bar{s}{ }^{12}$. Nos hallariamos por tanto ante el resto de un precioso arcaísmo del antiguo nominativo plural temático en $-\bar{o} s$ con caída de $-s$ final, conservado en una reducida parcela geográfica del Norte del Lacio.

La doctrina tradicional enseña que ${ }^{*}$-oi, marca de los nominativos plurales temáticos, es de origen pronominal ${ }^{13}$. A su vez ${ }^{*}-\bar{o} s$ sería la desinencia de los nombres. Esta teoría es gratuita y se basa en la prepotencia lingüistica del antiguo indio que durante mucho tiempo fue considerado como la lengua arquetipo para la reconstrucción del Indoeuropeo. Lo cierto es que el osco y el umbro sólo tienen $-\bar{o} s$. Asimismo el celta de la Península Ibérica emplea indiferentemente - $\bar{s}$ y $-o i$ en el nominativo plural y, por otro lado, el antiguo irlandés ha distribuido ambas desinencias entre nominativo y vocativo plural. Estos datos apun$\tan$ más bien hacia un fenómeno de alomorfismo en Indoeuropeo que hacia una temprana escisión de ambas desinencias en dos categorías, a saber, la nominal y la pronominal.

Anteriormente hemos adelantado que en IE. existian tres alomorfos para el nominativo plural temático, a saber, ${ }^{*}-\bar{o} s,{ }^{*}$-oi, ${ }^{*}{ }^{*}$ ois. Ninguno de ellos estaba adscrito exclusivamente a la categoria nominal ni a la pronominal. Cuando se fonologizó la oposición de cantidad, la desinencia $^{*}-\bar{o} s$ pudo marcar la categoria del plural, obteniendo así una forma diferenciada frente al nominativo singular y el primitivo genitivo singular $^{14}$.

Es verosimil que ${ }^{*}$-ois presentase también ${ }^{*}$-ois. Ahora bien estas nuevas formas indicarían simplemente un valor pluralizante expresando en un principio las funciones sintácticas de sujeto y complemento directo, y precisamente en antiguo indio tenemos restos de acusativos del plural en -äs en vez de -än que sería la forma esperada para la declina-

${ }^{12}$ Cf. A. Ernout, MSL 13, 1905-1906, y M. Leumann, Lateinische Grammatik Laut- und Formenlehre, Munich $1928_{5}$, p. 227.

${ }^{13} \mathrm{Cf}$., entre otros, A. Meillet, Historia de la lengua latina, Reus 1973, p. 22; P. Monteil, Éléments.... p. 163; W. Blümel, Untersuchungen.... p. 58. tivo.

14 La declinación temática hetita no distingue en el singular nominativo de geni- 
ción de los temas en ${ }^{*}-\bar{a}^{15}$. A estas formas de carácter pluralizante posteriormente se fueron añadiendo las desinencias regulares del plural, excepción hecha del acusativo que debió en seguida añadir a la vocal temática $-o$ la desinencia ${ }^{*}-n s<{ }^{*}-m+s$. Precisamente esta forma de acusativo plural sirvió también de base a las desinencias canónicas como puede verse en el genitivo plural de una gran parte de la declinación del antiguo indio: devắnām, dhīnắm, devínām etc... ${ }^{16}$.

Pues bien, tanto de ${ }^{*}-\bar{o} s$ como de ${ }^{*}$-oi y de ${ }^{*}-\overline{\bar{o}}$ is tenemos restos bien directamente como nominativos del plural o bien como formas bases a las que se añaden las desinencias, asi:

De *-ōs en el nominativo plural de nombres en antiguo indio y gótico, de nombres y pronombres en osco-umbro, alternando con -oi en el celta de la Península Ibérica y en función de vocativo en la declinación temática del antiguo irlandés y en los restos dialectales latinos anteriormente citados.

De forma indirecta podemos ver esta desinencia en el genitivo plural latino tipo deōrum $<{ }^{*}$ deōs-om.

De *-oi en el nominativo plural de los pronombres del antiguo indio $t e ́{ }^{*}{ }^{*} t o i$, en los pronombres y en los adjetivos fuertes del gótico: pai $<{ }^{*}$ toi, blindai $<{ }^{*}$-oi. En latín, griego y antiguo eslavo fue la desinencia generalizada tanto para nombres como para pronombres. En antiguo irlandés para el nominativo plural, así como para el celta de la Península Ibérica alternando con $-\bar{o} s$ Lutiacos y Lutiacei. En lenguas que tienen dual fue aprovechada para marcar el nom., voc. y ac. neutro del antiguo indio en la declinación temática de los nombres y pronombres, así yugé < ${ }^{*}$ yugoi, té < *toi e igualmente en antiguo eslavo donde se generalizó como podemos ver en ižěe, slovešě, imeně, otročentě y en sus pronombres oně, tě, etc...

De forma indirecta podemos verla en el dativo-ablativo plural del antiguo indio: devé-bhyas $<{ }^{*}$-oi-bhyos, tébhyas $<{ }^{*}$ toi-bhyos, en el dativo e instrumental del pronombre del antiguo eslavo: těmŭ, těmi < *oi$m o,{ }^{*}-o i-m i ̄$. Posiblemente también sobre esta forma se creó el gen.-loc.abl.-instr. dual que vemos en griego en -ouv, y en hom. -ouv.

De ${ }^{*}$-ois, como ya hemos señalado, tendríamos un resto en el nominativo plural temático latino en las formas acabadas en -es, -is, -eis. No obstante esta forma base puede entreverse en muchos casos de la declinación temática del plural de diversas lenguas: En el genitivo plural de

is Cf. F. Villar Liébana, Origen de la flexión nominal indoeuropea, Madrid 1974, p. 307.

16 También tenemos en ai. dévām como genitivo plural temático como en lat. deum. 
EM LVI 1, 1988

los pronombres masculinos y neutros del antiguo indio, téșām $<{ }^{*}$ tois$\bar{o} m$, en el genitivo plural de los adjetivos fuertes del gótico, blindaize < ${ }^{*}$ blindois-êm ${ }^{17}$, en el locativo y genitivo plural pronominal del antiguo eslavo téxŭ < ${ }^{*}$ tois-om, en el locativo plural del antiguo indio devéșu, téșu < *ois-u en el locativo plural de los nombres del antiguo eslavo raběxŭ $<{ }^{*}$ rabois- $u^{18}$ y en las formas griegas en -oıs y -oı en -oıs. El instrumental plural del antiguo indio se forma a partir de *ōis, asi como el dativo y ablativo plural latinos. Esta forma fue aprovechada una vez alargado el primer elemento del diptongo para la creación de estos $\operatorname{casos}^{19}$.

En resumen, si tenemos en cuenta que el hetita no posee la vocal temática $o$ en su nominativo y acusativo plural de la declinación temática, por cuanto presenta en el nominativo la marca pluralizante -es: antuhšeš y su acusativo es antuhšuš < *antuhs-ṇs, da la impresión de que la declinación temática del plural del indoeuropeo se fue creando paulatinamente en época postanatolia mediante un proceso aglutinativo consistente en añadir a las formas bases pluralizantes que contaban con tres alomorfos *-ōs, *-oi, *-ōis, las desinencias canónicas de los demás casos, si bien posteriormente o durante el mismo proceso pudieron existir otros procedimientos morfológicos. El latín, aunque generalizó para su nominativo plural temático la desinencia -oi, conserva algunos preciosos arcaísmos de $-\bar{o} s$ con caída de $-s$ en una reducida parcela del Norte del Lacio, y de *-ois en los nominativos plurales temáticos de algunos gentilicios acabados en -es, -is, -eis atestiguados en las inscripciones.

RAFAel JimÉnez Zamudio

\footnotetext{
17 También generalizado para el femenino: blindaizō $<{ }^{*}$-ois-ōm.

18 -ois más - $u$ marca de locativo. Cf. F. Villar Liébana, Origen..., p. 328.

19 Junto a deváis existe devébhis en ai. También el instrumental lituano descansa sobre *oōis > -ais y el del antiguo eslavo en $-y$ sobre *-ōis.
} 